\title{
Zinc oxide tetrapods as efficient photocatalysts for organic pollutant degradation
}

\author{
Fangzhou Liu ${ }^{\mathrm{a}}$, Yu Hang Leung ${ }^{\mathrm{a}}$, Aleksandra B. Djurišić*a ${ }^{\mathrm{a}}$, Changzhong Liao ${ }^{\mathrm{b}}$, Kaimin Shih ${ }^{\mathrm{b}}$ \\ ${ }^{a}$ Department of Physics, The University of Hong Kong, Pokfulam Road, Hong Kong; \\ ${ }^{b}$ Department of Civil Engineering, The University of Hong Kong, Pokfulam Road, Hong Kong
}

*dalek@hku.hk; phone +852-28597946

\begin{abstract}
Bisphenol A (BPA) and other organic pollutants from industrial wastewater have drawn increasing concern in the past decades regarding their environmental and biological risks, and hence developing strategies of effective degradation of BPA and other organic pollutants is imperative. Metal oxide nanostructures, in particular titanium oxide $\left(\mathrm{TiO}_{2}\right)$ and zinc oxide $(\mathrm{ZnO})$, have been demonstrated to exhibit efficient photodegradation of various common organic dyes. $\mathrm{ZnO}$ tetrapods are of special interest due to their low density of native defects which consequently lead to lower recombination losses and higher photocatalytic efficiency. Tetrapods can be obtained by relatively simple and low-cost vapor phase deposition in large quantity; the micron-scale size would also be advantageous for catalyst recovery. In this study, the photodegradation of $\mathrm{BPA}$ with $\mathrm{ZnO}$ tetrapods and $\mathrm{TiO}_{2}$ nanostructures under $\mathrm{UV}$ illumination were compared. The concentration of BPA dissolved in DI water was analyzed by high-performance liquid chromatography (HPLC) at specified time intervals. It was observed that the photocatalytic efficiency of $\mathrm{ZnO}$ tetrapods eventually surpassed Degussa P25 in free-standing form, and more than $80 \%$ of BPA was degraded after 60 min. Photodegradation of other organic dye pollutants by tetrapods and P25 were also examined. The superior photocatalytic efficiency of ZnO tetrapods for degradation of BPA and other organic dye pollutants and its correlation with the material properties were discussed.
\end{abstract}

Keywords: Zinc oxide, tetrapods, photodegradation

\section{INTRODUCTION}

Organic pollutants from industrial wastewater have long been a worldwide concern. Organic dyes form a common class of pollutants. In addition, endocrine disruptors, typically bisphenol A (BPA), have resulted in increasing risks for both marine environment and public health. ${ }^{1}$ Hence, efficient photocatalysts for degradation of the above pollutants are demanded. Titanium dioxide $\left(\mathrm{TiO}_{2}\right)$ has been extensively studied over the decades for its photocatalytic activity. It has been demonstrated that $\mathrm{TiO}_{2}$ is capable of degrading a wide range of organic pollutants in aqueous environment, including BPA. ${ }^{2,3}$ In addition to the two principal crystal phases, anatase and rutile, another commercially available form of $\mathrm{TiO}_{2}$, Degussa $\mathrm{P} 25$, has been widely applied for degradation of BPA and various organic dyes. ${ }^{3,4} \mathrm{P} 25$ consists of approximately $80 \%$ anatase and $20 \%$ rutile phase, and it exhibits higher photocatalytic activity than either pure phase. ${ }^{4}$ With the universally high photocatalytic activity, P25 is commonly used as a standard for photocatalytic studies. ${ }^{2-4}$

In addition to $\mathrm{TiO}_{2}$, other semiconducting materials such as zinc oxide $(\mathrm{ZnO})$ have drawn attention as potential candidates for high efficient photocatalysts. $\mathrm{ZnO}$ tetrapods exhibit unique structural and photocatalytic properties among various $\mathrm{ZnO}$ morphologies. ${ }^{5,6} \mathrm{ZnO}$ tetrapods can be synthesized by relatively simple and low-cost method in large quantity. Moreover, though with micron-scale morphology and inferior surface-to-volume ratio, $\mathrm{ZnO}$ tetrapods are reported to possess outstanding photocatalytic efficiency for degradation of several organic dyes. ${ }^{5,6}$ In this study, we compared the photocatalytic performance of $\mathrm{ZnO}$ tetrapods and $\mathrm{TiO}_{2} \mathrm{P} 25$ for degradation of BPA and selected dyes. The tetrapods exhibited better performance in all conditions studied. The attribution of superior photocatalytic performance of $\mathrm{ZnO}$ tetrapods was discussed in detail.

Oxide-based Materials and Devices VI, edited by Ferechteh H. Teherani, David C. Look, David J. Rogers, Proc. of SPIE Vol. 9364, 93641W · (C) 2015 SPIE · CCC code: 0277-786X/15/\$18 - doi: 10.1117/12.2078588 


\section{EXPERIMENTAL}

\subsection{Material preparation and characterization}

Degussa P25 was purchased from Evonik Industries. $\mathrm{ZnO}$ tetrapods were synthesized through a vapor-phase approach described as follows. ${ }^{5-7} 0.2 \mathrm{~g}$ of zinc powder $(99.995 \%$, Sigma Aldrich Co.) were placed in a quartz crucible inside a horizontal quartz tube and evaporated at $950{ }^{\circ} \mathrm{C}$ under $0.2 \mathrm{Lpm}$ humid argon flow. The white material was deposited on the downstream inner wall of the quartz tube and manually collected for further characterization. To examine the crystal and surface properties of $\mathrm{ZnO}$ tetrapods, high resolution transmission microscopy (HRTEM) and selected area electron diffraction (SAED) were performed using a JEOL JEM-2100F Field Emission Electron Microscope STEM. X-ray diffraction (XRD) patterns of $\mathrm{ZnO}$ tetrapods and Degussa P25 were measured with a Bruker D8 advance diffractometer $(\mathrm{Cu} \mathrm{K} \alpha$ radiation $(\lambda=0.154184 \mathrm{~nm})$ as the X-ray source). Fourier transform infrared spectroscopy (FTIR) measurements of the samples were performed using a SHIMADZU IRAffinity-1S Fourier Transform Infrared Spectrophotometer. For FTIR measurements, samples were well mixed into $\mathrm{KBr}$ (FT-IR grade, Sigma Aldrich Co.) and pressed to form transparent pellets using a $13 \mathrm{~mm}$ pellet-forming die. Background measurement was taken with a pellet of $\mathrm{KBr}$ only.

\subsection{Photodegradation of Bisphenol-A}

Bisphenol A (BPA, Sigma Aldrich Co.) was dissolved in deionized water at a concentration of $40 \mathrm{mg} / \mathrm{L} .50 \mathrm{mg}$ of the catalysts were mixed with $50 \mathrm{ml}$ of BPA solution in a Petri dish and stirred in dark for $30 \mathrm{~min}$ to reach equilibrium. The mixture was then subjected to UV illumination $\left(365 \mathrm{~nm}, 66.2 \mathrm{~mW} / \mathrm{cm}^{2}\right.$, Blak-Ray® B-100 AP Lamp). The BPA concentration was measured after specified exposure time $(0,15,30,45,60 \mathrm{~min})$ using a nanoscale reversed-phase highperformance liquid chromatography (HPLC, Agilent Technologies, Santa Clara, CA). The suspension was prefiltered with a Millex-FH PTFE filter $(0.45 \mu \mathrm{m}$ pore size $)$ to obtain a clear solution for HPLC measurements.

\subsection{Photodegradation of organic dyes}

5 common organic dyes were selected for photocatalytic experiments, including acid red 88 (International Lab USA), tropaeolin O (Acros Organics), cresyl violet acetate, naphthol blue black, and sunset yellow (Sigma Aldrich Co.). The dyes were dissolved in deionized water at a concentration of $10 \mathrm{mg} / \mathrm{L} .50 \mathrm{mg}$ of the catalysts were mixed with $50 \mathrm{ml}$ of dye solution in a Petri dish and stirred in dark for $30 \mathrm{~min}$, following by exposure to UV illumination. Absorption spectra were measured using a PerkinElmer Lambda Bio 40 UV/VIS spectrometer immediately before exposure to UV illumination and at specified time intervals during UV exposure. For each measurement, $3 \mathrm{ml}$ of the suspension was withdrawn and filtered to obtain a clear solution.

\section{RESULTS AND DISCUSSION}

The high-resolution TEM image and SAED pattern of a tetrapod leg in Figure 1 illustrate excellent crystal properties of the material. The legs have a wurtzite structure with a growth direction along [0001] and (10 $\overline{1} 0)$ terminating facets. It is noted that the nonpolar $(10 \overline{1} 0)$ surface has been reported to exhibit the highest photocatalytic activity and stability among different crystallographic orientations of the ZnO crystal. ${ }^{8}$ The XRD patterns in Figure 2 also confirm the high crystallinity of the $\mathrm{ZnO}$ tetrapods. The sharp diffraction peaks in the XRD pattern of tetrapods match the characteristic $\mathrm{ZnO}$ wurtzite structure, with no other crystal phases observed, in agreement with literature. ${ }^{5,6}$ 


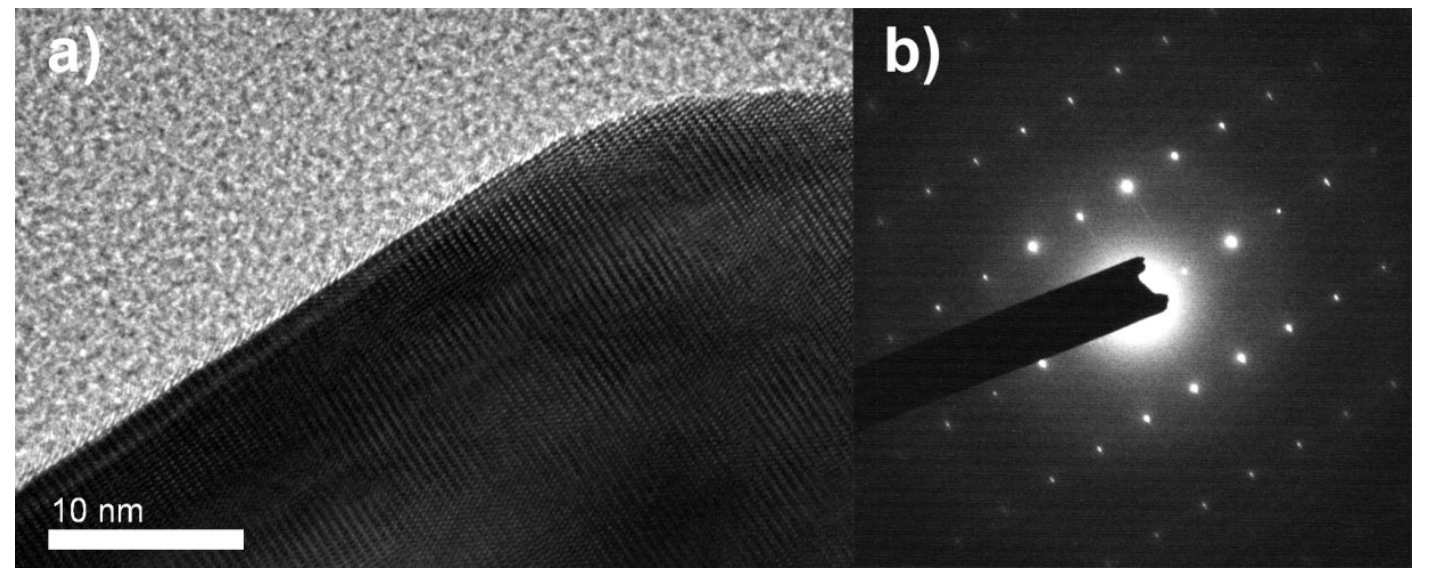

Figure 1. a) HRTEM image and b) SAED pattern of $\mathrm{ZnO}$ tetrapods.

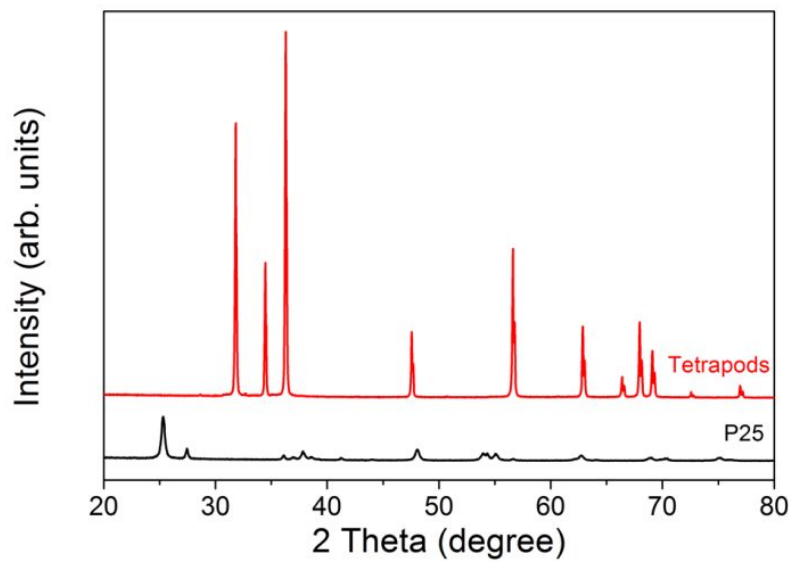

Figure 2. XRD patterns of $\mathrm{ZnO}$ tetrapods and Degussa P25.

Figure 3 shows the FTIR spectra of ZnO tetrapods and P25 nanoparticles. Except the broad resonance at $\sim 3200-3600$ $\mathrm{cm}^{-1}$ corresponding to $\mathrm{OH}$ group vibration and the peak at $\sim 1630 \mathrm{~cm}^{-1}$ assigned to the scissoring mode of the molecular water, ${ }^{9}$ there is no significant peak identified in the spectra for both samples, indicating negligible presence of organic surface adsorbates.

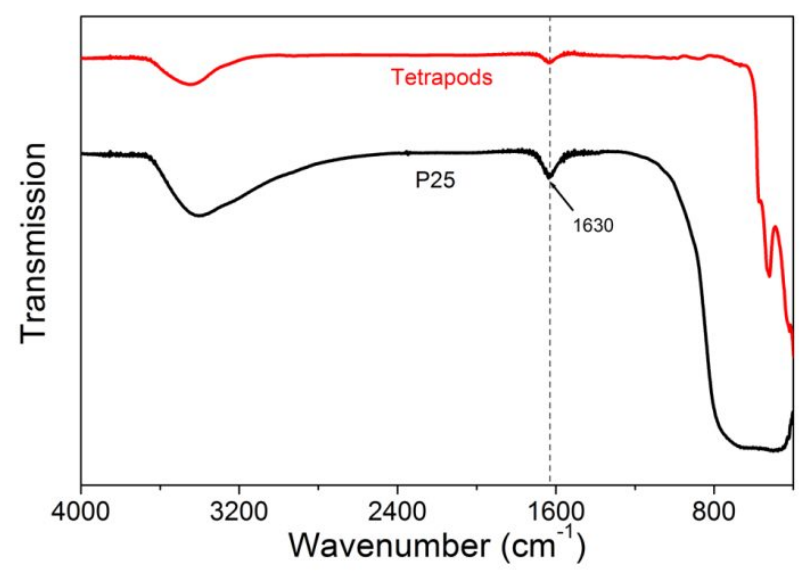

Figure 3. FTIR spectra of ZnO tetrapods and Degussa P25. 
Figure 4 shows the photodegradation of BPA by ZnO tetrapods and P25. Despite that P25 exhibits faster degradation rate during the initial $20 \mathrm{~min}$, the photocatalytic performance of $\mathrm{ZnO}$ tetrapods eventually surpasses P25 as the UV exposure time increases. After 60 min of UV irradiation, more than $80 \%$ of BPA is degraded with tetrapods as the photocatalyst, indicating excellent photocatalytic efficiency of tetrapods towards BPA. Figure 5 summarizes the photodegradation curves of different organic dyes by $\mathrm{ZnO}$ tetrapods and P25. For all dyes selected, tetrapods result in comparable or higher photocatalytic performance over P25, which provides a support to the universal remarkable photoactivity of $\mathrm{ZnO}$ tetrapods.

The results in this study, together with other reports in the literature, ${ }^{5,10}$ demonstrate the superior photocatalytic performance of $\mathrm{ZnO}$ tetrapods for a variety of organic pollutants compared to Degussa P25 which is commonly regarded as a standard, highly efficient photocatalyst. Such high performance of tetrapods may stem from their intrinsic material properties. It has been reported that $\mathrm{ZnO}$ tetrapods exhibits excellent optical properties, for instance long photoluminescene (PL) lifetime at specified growth temperature, high UV-to-defect emission ratio, or even with the absence of visible defect emission in the room temperature photoluminescence. ${ }^{7} \mathrm{ZnO}$ tetrapods also possess excellent crystal quality with relatively low native defect concentration. ${ }^{5-7}$ Native defects have been reported to play a hindering role in the photocatalysis reaction since they serve as the recombination center of the photogenerated electron-hole pairs and hence lead to recombination losses. ${ }^{5}$ Thus lower native defects would contribute to higher photocatalytic efficiency. On the other hand, smooth, clean surfaces with negligible surface adsorbates, as well as a higher ratio of smooth $(10 \overline{1} 0)$ facets which exhibit high photocatalytic activity, ${ }^{8}$ all these morphological advantages favor photocatalytic efficiency of $\mathrm{ZnO}$ tetrapods. One point worth noticing is that $\mathrm{ZnO}$ tetrapods have a micron-scale size and siginificantly lower surface area compare to Degussa P25 nanoparticles and other nanostructured photocatalysts. High surface area is conventionally considered as a crucial factor enhancing photocatalytic efficiency. ${ }^{11}$ However, in the case of $\mathrm{ZnO}$ tetrapods, superior performance is observed over a range of different $\mathrm{ZnO}$ and $\mathrm{TiO}_{2}$ nanoparticles with higher surface area than tetrapods. This may indicate that the excellent crystal and structural properties rather than surface area play a more determining role in the photoactivity of $\mathrm{ZnO}$ tetrapods.

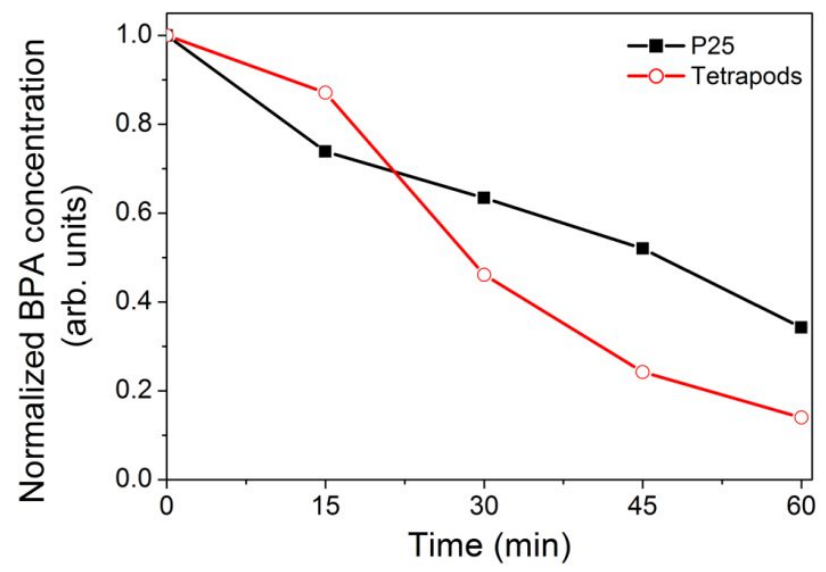

Figure 4. Photodegradation curve of BPA aqueous solution by $\mathrm{ZnO}$ tetrapods and Degussa P25. 

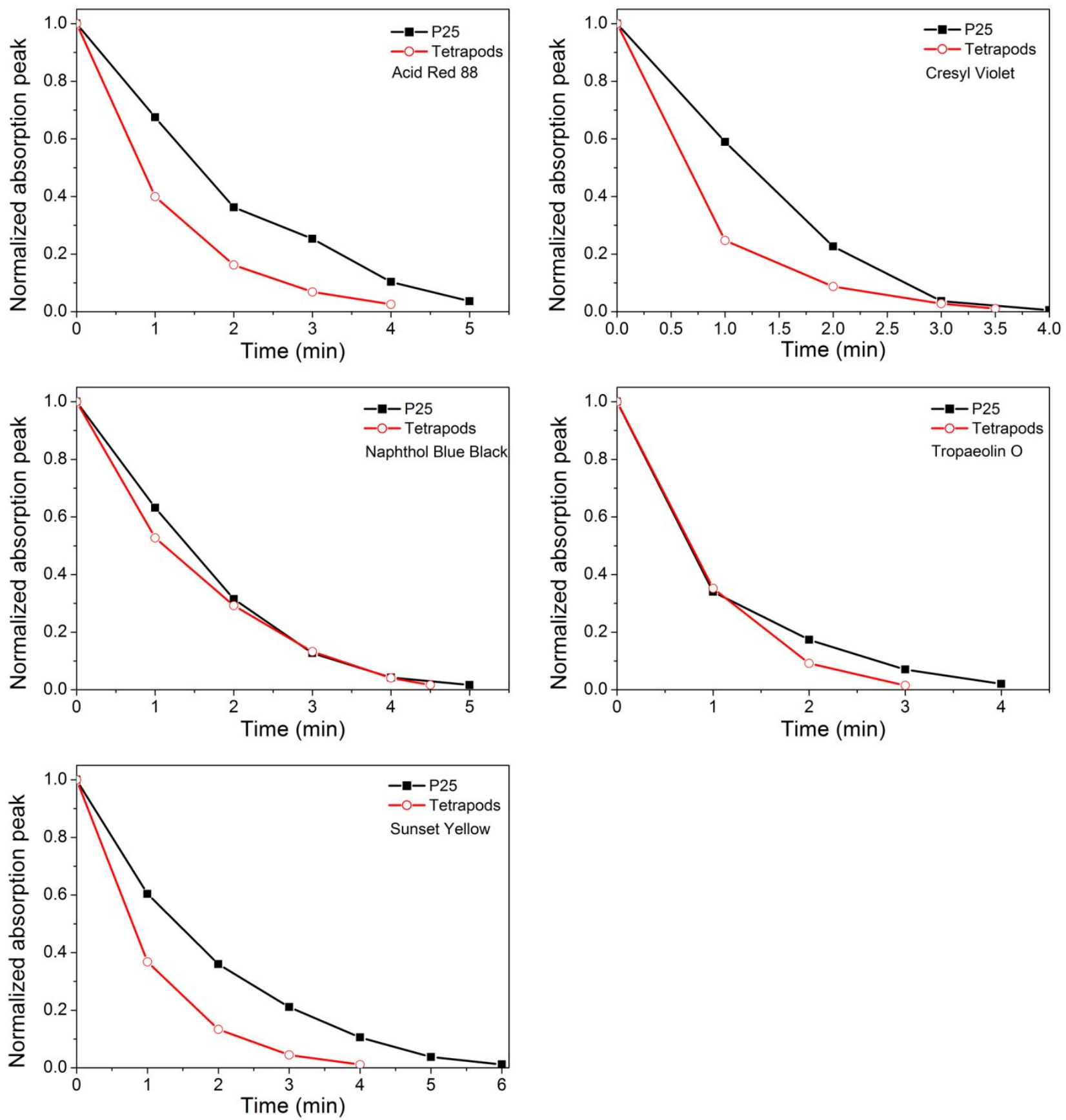

Figure 5. Photodegradation curves of different dyes by $\mathrm{ZnO}$ tetrapods and Degussa P25. 


\section{CONCLUSION}

In this study we examined the photocatalytic activity of vapor-phase synthesized $\mathrm{ZnO}$ tetrapods compared with commercially available Degussa P25 $\mathrm{TiO}_{2}$ nanoparticles as the standard catalyst. $\mathrm{ZnO}$ tetrapods exhibit higher performance in photodegradation of bisphenol A and various organic dyes than P25. The high crystallinity, smooth surface with specific photoactive terminating facets, as well as the negligible presence of surface adsorbates contribute to the superior photocatalytic activity of $\mathrm{ZnO}$ tetrapods.

\section{ACKNOWLEDGEMENT}

The authors would like to thank Dr. Jill Man Ying Chiu and Miss Gege Zhao from Hong Kong Baptist University for their help with HPLC measurements.

\section{REFERENCES}

[1] Kondrakov, A. O., Ignatev, A. N., Frimmel, F. H., Bräse, S., Horn, H. and Revelsky, A. I., "Formation of genotoxic quinones during bisphenol A degradation by $\mathrm{TiO}_{2}$ photocatalysis and UV photolysis: A comparative study," Appl. Catal., B 160-161, 106-114 (2014).

[2] Ohko, Y., Ando, I., Niwa, C., Tatsuma, T., Yamamura, T., Nakashima, T., Kubota, Y. and Fujishima, A., "Degradation of bisphenol A in Water by $\mathrm{TiO}_{2}$ photocatalyst," Environ. Sci. Technol. 35, 2365-2368 (2001).

[3] Chiang, K., Lim, T. M., Tsen, L. and Lee, C. C., "Photocatalytic degradation and mineralization of bisphenol A by $\mathrm{TiO}_{2}$ and platinized $\mathrm{TiO}_{2}$, , Appl. Catal. A 261, 225-237 (2004).

[4] Woan, K., Pyrgiotakis, G. and Sigmund, W., "Photocatalytic carbon-nanotube- $\mathrm{TiO}_{2}$ composites," Adv. Mater. 21, 2233-2239 (2009).

[5] Guo, M. Y., Ng, A. M. C., Liu, F. Z., Djurišić, A. B., Chan, W. K., Su, H. M. and Wong, K. S., "Effect of native defects on photocatalytic properties of ZnO," J. Phys. Chem. C 115, 11095-11101 (2011).

[6] Liu, F. Z., Leung, Y. H., Djurišić, A. B., Ng, A. M. C., Chan, W. K., Ng, K. L., Wong, K. S., Liao, C. Z., Shih, K. M. and Surya, C., "Effect of Plasma Treatment on Native Defects and Photocatalytic Activities of Zinc Oxide Tetrapods," J. Phys. Chem. C 118 (39), 22760-22767 (2014).

[7] Zhong, Y. C., Djurišić, A. B., Hsu, Y. F., Wong, K. S., Brauer, G., Ling, C. C. and Chan, W. K., "Exceptionally long excitation photoluminescence lifetime in ZnO tetrapods," J. Phys. Chem. C 112, 16286-16295 (2008).

[8] Kislov, N., Lahiri, J., Verma, H., Goswami, D. Y., Stefenakos, E. and Batzill, M. "Photocatalytic degradation of methyl orange over single crystalline $\mathrm{ZnO}$ : Orientation dependence of photoactivity and photostability of $\mathrm{ZnO}$," Langmuir 25, 3310-3315 (2009).

[9] Noei, H., Qiu, H. S., Wang, Y. M., Löffler, E., Wöll, C. and Muhler, M., "The identification of hydroxyl groups on ZnO nanoparticles by infrared spectroscopy," Phys. Chem. Chem. Phys. 10, $7092-7097$ (2008).

[10] Wan, Q., Wang, T. H. and Zhao, J. C., "Enhanced photocatalytic activity of ZnO nanotetrapods," Appl. Phys. Lett. 87, 083105 (2005).

[11] Xu, F., Yuan, Z. Y., Du, G. H., Ren, T. Z., Bouvy, C., Halasa, M., Su, B. L., "Simple approach to highly oriented $\mathrm{ZnO}$ nanowire arrays: Large-scale growth, photoluminescence and photocatalytic properties," Nanotechnology 17, 588-594 (2006). 